PROCEEDINGS OF THE

AMERICAN MATHEMATICAL SOCIETY

Volume 136, Number 10, October 2008, Pages 3415-3418

S 0002-9939(08)09449-5

Article electronically published on May 16, 2008

\title{
A $2 \times 2$ LATTICE SPACE-TIME CODE OF RANK 5
}

\author{
CHAOPING XING AND WEI LI
}

(Communicated by Wen-Ching Winnie Li)

\begin{abstract}
For all previous constructions of $2 \times 2$ lattice space-time codes with a positive diversity product, the rank was at most 4 . In this paper, we give an example of a $2 \times 2$ lattice space-time code of rank 5 with a positive diversity product.
\end{abstract}

\section{INTRODUCTION}

In the recent years, there has been a lot of research on space-time codes. Many mathematical subjects such as number theory, algebra, combinatorics, etc., have been employed to construct good lattice codes [1, 2, 3, 5, 6, 7.

Denote by $\mathcal{M}_{n}(\mathbb{C})$ the set of $n \times n$ matrices over $\mathbb{C}$. A lattice space-time code over $\mathbb{C}$ is a set $\mathcal{A}$ consisting of matrices in $\mathcal{M}_{n}(\mathbb{C})$ such that $\mathcal{A}$ is a free abelian group under the matrix addition. The rank of this group is called the dimension or rank of $\mathcal{A}$.

There are some parameters to measure a lattice space-time code.

Criteria for lattice space-time codes:

(i) the rank of $\mathcal{A}$ should be as large as possible;

(ii) the diversity product defined by

$$
\delta(\mathcal{A}):=\inf \{|\operatorname{det}(A-B)|: A, B \in \mathcal{A}, A \neq B\}
$$

should be as large as possible, where $|\cdot|$ stands for the absolute value of a real number or complex number;

(iii) the discriminant of $\mathcal{A}$ should be as small as possible (see the definition of the discriminant of a complex lattice below).

A natural question is:

what is the maximal rank of a lattice space-time code $\mathcal{A}$ such that $\delta(\mathcal{A})>0$ ? In other words, we want to determine

$$
r(n):=\max \left\{\operatorname{rank}(\mathcal{A}): \mathcal{A} \text { is a lattice in } \mathcal{M}_{n}(\mathbb{C}), \delta(\mathcal{A})>0\right\} .
$$

Determining the exact value for $r(n)$ seems difficult. One can imagine that it is also not easy to give some reasonable bounds. One obvious lower bound is $r(n) \geq 2 n$ (see [8, 1, 2, 6, 7). In this paper, we give an upper bound, i.e., $r(n) \leq 2 n^{2}$.

Received by the editors August 24, 2007.

2000 Mathematics Subject Classification. Primary 06B99, 15A15, 11Z05, $11 \mathrm{H} 71$.

Key words and phrases. Rank, diversity product, lattice.

The first author is supported by Singapore MDE-ARF research grant R60/07 and National Scientific Research Project 973 of the People's Republic of China, 2004CB318000.

(C)2008 American Mathematical Society Reverts to public domain 28 years from publication 
The case $n=2$ is of particular interest. However, even in this case, we only know $r(n) \geq 4$, and so far we have not constructed any $2 \times 2$ lattice space-time codes of rank larger than 4 with a positive diversity product. In this paper, we produce an example of a $2 \times 2$ lattice space-time code of rank 5 with a positive diversity product.

\section{An upper bound on RANK of Lattice SPACE-Time CODES}

The main purpose of this section is to give an upper bound on $r(n)$.

Lemma 2.1. Let $\mathcal{A}$ be a lattice space-time code in $\mathcal{M}_{n}(\mathbb{C})$. If the diversity product $\delta(\mathcal{A})>0$, then the rank of $\mathcal{A}$ is at most $2 n^{2}$. Therefore, $r(n) \leq 2 n^{2}$.

Proof. We can identify the space $\mathcal{M}_{n}(\mathbb{C})$ with $\mathbb{C}^{n^{2}}$. Thus, we introduce the usual distance in $\mathcal{M}_{n}(\mathbb{C})$ defined by

$$
d(A, B)=\sqrt{\sum_{i, j}\left|a_{i j}-b_{i j}\right|^{2}}
$$

for two matrices $A=\left(a_{i j}\right)$ and $B=\left(b_{i j}\right)$ in $\mathcal{M}_{n}(\mathbb{C})$. It is clear that we have the obvious inequality.

$$
\begin{aligned}
|d(A, B)| & =\left(\sum_{i=1}^{n} \sum_{j=1}^{n}\left|a_{i j}-b_{i j}\right|^{2}\right)^{1 / 2} \\
& \geq\left(n\left(\prod_{i=1}^{n} \sum_{j=1}^{n}\left|a_{i j}-b_{i j}\right|^{2}\right)^{1 / n^{2}}\right)^{1 / 2} \\
& =\sqrt{n}|\operatorname{det}(A-B)|^{1 / n^{2}}
\end{aligned}
$$

Hence, we have

$$
|\operatorname{det}(A-B)| \leq n^{-n^{2} / 2}|d(A, B)|^{n^{2}} .
$$

Let $\left\{A_{1}, \ldots, A_{t}\right\}$ be a $\mathbb{Z}$-basis with $t=\operatorname{rank}(\mathcal{A})$. Then $\left\{A_{1}, \ldots, A_{t}\right\}$ is linearly independent over $\mathbb{Q}$.

We conclude that $\left\{A_{1}, \ldots, A_{t}\right\}$ is linearly independent over $\mathbb{R}$ as well. Suppose that $\left\{A_{1}, \ldots, A_{t}\right\}$ is linearly dependent over $\mathbb{R}$. Then one of the matrices is a $\mathbb{R}$-linear combination of others. We may assume that

$$
A_{t}=\sum_{i=1}^{t-1} \alpha_{i} A_{i} \quad \text { for some } \alpha_{i} \in \mathbb{R} .
$$

Define the set

$$
\mathcal{T}:=\left\{\sum_{i=1}^{t-1} a_{i} A_{i}: 0 \leq a_{i}<1,1 \leq i \leq t-1\right\} .
$$

Then $\mathcal{T}$ is a bounded domain. For each $m \geq 1$, by (2.3), we have

$$
m A_{t}=\sum_{i=1}^{t-1} m \alpha_{i} A_{i}=\sum_{i=1}^{t-1} c_{i} A_{i}+\sum_{i=1}^{t-1} b_{i} A_{i}
$$


with $c_{i}=m \alpha_{i}-\left\lfloor m \alpha_{i}\right\rfloor$ and $b_{i}=\left\lfloor m \alpha_{i}\right\rfloor$. Put $B_{m}=\sum_{i=1}^{t-1} b_{i} A_{i}$ and $C_{m}=\sum_{i=1}^{t-1} c_{i} A_{i}$. Then we have $B_{m} \in \mathcal{A}$ and $C_{m} \in \mathcal{T}$. Consider the sequence $\left\{m A_{t}-B_{m}=\right.$ $\left.C_{m}\right\}_{m=1}^{\infty} \subset \mathcal{A} \cap \mathcal{T}$. It is clear that all matrices in the sequence are distinct, as $\left\{A_{1}, \ldots, A_{t}\right\}$ is linearly independent over $\mathbb{Z}$.

Since this sequence is in the bounded domain $\mathcal{T}$, we can find a matrix $D \in$ $\mathcal{M}_{n}(\mathbb{C})$ such that there is a subsequence $\left\{C_{m_{i}}\right\}_{i=1}^{\infty}$ with a limit

$$
\lim _{i \rightarrow \infty} C_{m_{i}}=D \text {. }
$$

Thus, for any $\varepsilon>0$, there exists $N$ such that $\left|d\left(C_{m_{N}}, C_{m_{N+1}}\right)\right| \leq \sqrt{n} \varepsilon^{1 / n^{2}}$. Hence, by (2.2) we have $\left|\operatorname{det}\left(C_{m_{N}}-C_{m_{N+1}}\right)\right| \leq \varepsilon$. This implies that $\delta(\mathcal{A})=0$. This contradiction means that $\left\{A_{1}, \ldots, A_{t}\right\}$ is linearly independent over $\mathbb{R}$. The desired result follows from the fact that

$$
t=\operatorname{rank}(\mathcal{A})=\operatorname{dim}_{\mathbb{R}}\left(\mathcal{A}_{\mathbb{R}}\right) \leq \operatorname{dim}_{\mathbb{R}}\left(\mathcal{M}_{n}(\mathbb{C})\right)=2 n^{2},
$$

where $\mathcal{A}_{\mathbb{R}}:=\left\{\sum_{i=1}^{t} \alpha_{i} A_{i}: \quad \alpha_{i} \in \mathbb{R}\right\}$ is the linear space over $\mathbb{R}$ generated by $\left\{A_{1}, \ldots, A_{t}\right\}$.

\section{Construction}

We need the following result from elementary number theory for this section (see 4. Th. 3 of p. 391]).

Lemma 3.1. A positive integer can be expressed as a sum of three squares of integers only if it is not of the form $4^{r}(8 t+7)$ for some integers $r, t \geq 0$.

Consider the five $5 \times 5$ matrices over $\mathbb{C}$ :

$$
A_{1}=\left(\begin{array}{cc}
1 & 0 \\
0 & 1
\end{array}\right) ; \quad A_{2}=\left(\begin{array}{cc}
i & 0 \\
0 & -i
\end{array}\right) ; \quad A_{3}=\left(\begin{array}{cc}
0 & 1 \\
-1 & 0
\end{array}\right) ;
$$

and

$$
A_{4}=\left(\begin{array}{cc}
0 & i \\
i & 0
\end{array}\right) ; \quad A_{5}=\left(\begin{array}{cc}
\sqrt{7} & 0 \\
0 & -\sqrt{7}
\end{array}\right) .
$$

Theorem 3.2. Let $\mathcal{A}$ be the lattice generated by the above five matrices. Then the rank of $\mathcal{A}$ is 5 and the diversity product of $\mathcal{A}$ is 1 .

Proof. First of all, we have $\operatorname{det}\left(A_{1}\right)=1$. Hence, the diversity product of $\mathcal{A}$ is at most 1.

To show that the rank of $\mathcal{A}$ is 5 and the diversity product of $\mathcal{A}$ is at least 1 , it is sufficient to prove that for any five integers $a, b, c, d, e$ with $(a, b, c, d, e) \neq$ $(0,0,0,0,0)$ we have $|\operatorname{det}(A)| \geq 1$ with $A=a A_{1}+b A_{2}+c A_{3}+d A_{4}+e A_{5}$.

It is easy to verify that

$$
\begin{aligned}
|\operatorname{det}(A)| & =\left|a^{2}+b^{2}+c^{2}+d^{2}-7 e^{2}-2 \sqrt{7} b e i\right| \\
& =\sqrt{\left(a^{2}+b^{2}+c^{2}+d^{2}-7 e^{2}\right)^{2}+28 b^{2} e^{2}} .
\end{aligned}
$$

We can show $|\operatorname{det}(A)| \geq 1$ by distinguishing several cases:

(i) If $b e \neq 0$, then we have $|\operatorname{det}(A)| \geq \sqrt{28}$.

(ii) If $e$ is equal to 0 , then $|\operatorname{det}(A)|=\sqrt{a^{2}+b^{2}+c^{2}+d^{2}} \geq 1$. 
(iii) Finally we assume that $b=0$; then $|\operatorname{det}(A)|^{2}=\left|a^{2}+c^{2}+d^{2}-7 e^{2}\right|$. Write $e$ into the form $2^{r} f$ with $f$ being an odd integer. Then $7 e^{2}=4^{r}\left(7 f^{2}\right)=$ $4^{r}(8 t+7)$ for some $t \geq 0$. By Lemma 3.1. $7 e^{2}$ cannot be equal to $a^{2}+c^{2}+d^{2}$. Thus, $|\operatorname{det}(A)|^{2}=\left|a^{2}+c^{2}+d^{2}-7 e^{2}\right|>0$. Since $|\operatorname{det}(A)|^{2}$ is an integer, we have $|\operatorname{det}(A)|^{2} \geq 1$.

This completes the proof.

Remark 3.3. For our lattice $\mathcal{A}$ in Theorem 3.2 the dimension of the tensor product $\mathcal{A} \otimes_{\mathbb{Q}} \mathbb{R}$ has dimension 5 over $\mathbb{R}$. Although there exists a $2 \times 2$ lattice $\mathcal{B}$ of rank 8 such that the determinant of difference of every two distinct matrices in $\mathcal{B}$ is not vanishing, its inferior limit is still equal to 0 . As far as we know, the lattice in this paper is the first $2 \times 2$ one of rank larger than 4 with a positive inferior limit.

By Lemma 2.1. we know that a $2 \times 2$ lattice space-time code with positive diversity is at most 8 , and our Theorem 3.2 shows the existence of a $2 \times 2$ lattice space-time code of rank 5 with a positive diversity product. This raises the following question.

Open Problem. Is there a $2 \times 2$ lattice space-time code of rank bigger than 5 with a positive diversity product?

\section{ACKNOWLEDGEMENT}

The authors are grateful to Dr. L. J. Xu for some useful comments.

\section{REFERENCES}

[1] M. O. Damen, A. Tewfik and J.-C. Belfiore, A construction of a space-time code based on number theory, IEEE Trans. Inform. Theory, Vol. 48, No. 3(2002), 753-760. MR1889980 (2003i:94003)

[2] P. Dayal and M. K. Varanasi, An algebraic family of complex lattices for fading channels with application to space-time codes, IEEE Trans. Inform. Theory, Vol. 51, No. 12(2005), 4184-4202. MR2243151 (2007b:94153)

[3] A. Shokrollahi, B. Hassibi, B. M. Hochwald and W. Sweldens, Representation theory for high-rate multiple-antenna code design, IEEE Trans. Inform. Theory, Vol. 47, No. 6(2001), 2335-2367. MR:1873925 (2003j:94120)

[4] W. Sierpiński, Elementary Theory of Numbers, North-Holland, 1964. MR0175840 (31:116)

[5] V. Tarokh, H. Jafarkhani and A. R. Calderbank, Space-time block codes from orthogonal designs, IEEE Trans. Inform. Theory, Vol. 45, No. 5(1999), 1456-1467. MR1699070 (2000g:94001)

[6] G. Wang, H. Liao, H. Wang, and X.-G. Xia, Systematic and optimal cyclotomic lattices and diagonal space-time block code designs, IEEE Trans. Inform. Theory, Vol. 50, No. 12(2004), pp. 3348-3360. MR 2103509

[7] G. Wang and X.-G. Xia, On optimal multilayer cyclotomic space-time code designs, IEEE Trans. Inform. Theory, Vol. 51, No. 3(2005), pp. 1102-1135. MR2237973 (2007h:94088a)

[8] C. P. Xing, Diagonal Lattice Space-Time Codes from Number Fields and Asymptotic Bounds, IEEE Trans. Inform. Theory, Vol. 53, No. 11(2006), pp. 3921-3926.

Division of Mathematical Science, School of Physical and Mathematical Sciences, Nanyang Technological University, Singapore 637616, Republic of Singapore

E-mail address: xingcp@ntu.edu.sg

Department of Mathematical Sciences, Tsinghua University, Bejing, People's RepubLIC OF CHINA 